\title{
Change of carrier spectrum in layer semiconductors caused by strong electromagnetic wave field
}

\author{
B.A. Lukiyanets \\ National University "Lvivs'ka Politekhnika", 12 Bandera St., 79646 Lviv, Ukraine \\ Phone: +38(0322) 34 5139; e-mail: lukbog@ua.fm
}

\begin{abstract}
Spectrum of carriers in layer semiconductors under action of strong electromagnetic field is analyzed. It is shown that obtained modification of the spectrum qualitatively differs from modification in analogous problem in excitonic dielectric or superconductor with the parabolic dispersion law of carriers.
\end{abstract}

Keywords: layer crystal, electromagnetic field, electron-photon interaction, electron states.

Paper received 22.05.03; accepted for publication 16.06.03.

\section{Introduction}

Aim of the paper is analysis of an electron spectrum of the sharply anisotropic semiconductors in the field of strong electromagnetic radiation. Phenomenological model is useless for the description the problem because its some active constraints, and it needs the microscopic description [1].

Let us consider layer semiconductors. It is known [2] they possess by sharply anisotropic physical properties caused by various type of chemical bondings (covalent or ion-covalent and van der Waals) in various their crystallographic directions. Let us the layers coincide with the XOY planes. Then the electron and hole dispersion laws take the form

$\varepsilon_{c}(\vec{p})=\alpha_{c} p_{\perp}^{2}+\beta_{c}(1-\cos z)+\frac{E_{g}}{2}$

$\varepsilon_{h}(\vec{p})=\alpha_{h} p_{\perp}^{2}+\beta_{h}(1-\cos z)+\frac{E_{g}}{2}$

where $p_{\perp}=\left(p_{x}, p_{y}\right), \alpha_{c}=\frac{1}{2 m_{c}^{*}}, \alpha_{h}=\frac{1}{2 m_{h}^{*}}\left(m_{c}^{*}, m_{h}^{*}\right.$ are effective masses of an electron and a hole, respectively; $\hbar=1) ; \beta_{c}, \beta_{h}$ are the electron and hole mixings, respectively; $z=k_{z} d_{z}$. The origin is taken in the middle of forbidden energy gap $E_{g}$.

Description of the carriers in (1), (2) in the effective mass approximation in the plane of layer and in the tightbinding approximation normally to it shows noted peculiarities of the layer crystals.

Let us insert the layer semiconductor into the field of the strong running electromagnetic wave

$E(\bar{r}, t)=E \exp (i \Omega t+i \varphi)$,

where $\Omega$ is frequency of the wave. Since we will consider resonance interaction of such field with the semiconductor, the frequency must satisfy the condition [3]

$0<\Omega-E_{g} \ll E_{g}$.

\section{Hamiltonian of the problem. Green function method of its solution}

Hamiltonian which describes the carriers of the semiconductor in the electromagnetic field in the secondary quantization representation takes the form [1]

$$
\begin{aligned}
& H(t)=\sum_{\vec{p}}\left\{\varepsilon_{c}(\vec{p}) a_{\vec{p}}^{+} a_{\vec{p}}+\varepsilon_{h}(\vec{p}) b_{\vec{p}}^{+} b_{\vec{p}}\right\}+ \\
& +\sum_{\vec{p} \underline{p}^{\prime}}\left\{\lambda_{\vec{p} \vec{p}^{\prime}} a_{\vec{p}}^{+} b_{\vec{p}^{\prime}}^{+} \exp (-i \Omega t)+\text { h.c. }\right\}
\end{aligned}
$$




\section{B.A. Lukiyanets: Change of carrier spectrum in layer semiconductors ...}

Here $a_{\vec{p}}^{+}\left(b_{\vec{o}}^{+}\right)$is a creation operator, and a $a_{\vec{p}}\left(b_{\vec{o}}\right)$ is an annihilation operator of an electron (hole), respectively.

The last term in (5) describes interaction with electromagnetic field, where $a_{\vec{p}}^{+} b_{\vec{p}^{\prime}}^{+}$describes the electron transfer from valence band to conduction band, and $a_{\vec{p}} b_{\vec{p}^{\prime}}$ reverse process. Matrix element $\lambda_{\vec{p} \cdot \vec{p}}$ may be presented as

$\lambda_{\vec{p}^{\prime} \vec{p}}=\lambda \delta_{\vec{p}^{\prime} \vec{p}}$

where

$\lambda=E_{\vec{p} \vec{p}} d_{c v}$

and

$$
\begin{aligned}
& E_{\vec{p}^{\prime} \vec{p}}=\int E(\vec{r}) \exp \left[i\left(\vec{p}-\vec{p}^{\prime}\right) \vec{r}\right] d^{3} r, \\
& d_{c v}=\frac{e V_{c v}}{\Omega}(c=1) .
\end{aligned}
$$

Matrix element $\lambda_{\vec{p} \cdot \vec{p}}$ connects two states $\vec{p}$ and $\vec{p}^{\prime}$ in $c$-and $v$-bands. Since $\vec{p}-\vec{p}^{\prime}=\vec{k}$ - wave vector of the electromagnetic field is much less than quasi-momentum of the electron $\left(|\vec{k}| \approx 10^{4} \mathrm{~cm}^{-1}\right.$, a $\left.|\vec{p}| \approx 10^{6} \mathrm{~cm}^{-1}[1,3]\right)$, it may be ignored. Taking into account this fact,

$$
\begin{aligned}
& H(t)=\sum_{\vec{p}}\left\{\varepsilon_{c}(\vec{p}) a_{\vec{p}}^{+} a_{\vec{p}}+\varepsilon_{h}(\vec{p}) b_{\vec{p}}^{+} b_{\vec{p}}\right\}+ \\
& +\sum_{\vec{p}}\left\{\lambda_{\vec{p} \vec{p}} a_{\vec{p}}^{+} b_{\vec{p}}^{+} \exp (-i \Omega t)+\text { h.c. }\right\}
\end{aligned}
$$

By unitary transformation proposed in [4]

$$
U(t)=\exp \left\{-\frac{i \Omega t}{2} \sum_{\vec{p}}\left(a_{\vec{p}}^{+} a_{\vec{p}}+b_{\vec{p}}^{+} b_{\vec{p}}\right)\right\}
$$

Hamiltonian (9) may be presented in the form independent on time, namely:

$$
\begin{aligned}
& H=\sum_{\vec{p}}\left\{\xi_{c}(\bar{p}) a_{\vec{p}}^{+} a_{\vec{p}}+\xi_{h}(\vec{p}) b_{\vec{p}}^{+} b_{\vec{p}}\right\}+ \\
& +\sum_{\vec{p}}\left\{\lambda_{\vec{p}} \vec{p} a_{\vec{p}}^{+} b_{\vec{p}}^{+}+\text {h.c. }\right\}
\end{aligned}
$$

where $\xi_{c}(\vec{p})=\varepsilon_{c}(\vec{p})-\mu_{0}, \xi_{v}(\vec{p})=\varepsilon_{v}(\vec{p})-\mu_{0}$ with $\mu_{0}=$

$$
=\frac{\Omega-E_{g}}{2} \text {. }
$$

Thus the unitary transformation leads to the shift $\mu_{0}$ of the bands and as result according to condition (4) the bands will be intersect.

Structure of the Hamiltonian (11) looks like to the Hamiltonian which describes superconductivity or excitonic dielectric [4]. It is known that the Hamilto- nian may be diagonalized by alternative methods. One of them is using Green function method.

Let us introduce Green functions [4]

$G_{a}\left(\vec{p} t ; \vec{p}^{\prime} t^{\prime}\right)=-i<T a_{\vec{p}}(t) a_{\vec{p}^{\prime}}^{+}\left(t^{\prime}\right)>$

$G_{b}\left(\vec{p} t ; \vec{p}^{\prime} t^{\prime}\right)=-i<T b_{\vec{p}}(t) b_{\vec{p}^{\prime}}^{+}\left(t^{\prime}\right)>$

$F^{+}\left(\vec{p} t ; \vec{p}^{\prime} t^{\prime}\right)=<T b_{\vec{p}}^{+}(t) a_{\vec{p}^{\prime}}^{+}\left(t^{\prime}\right)>$

$F\left(\vec{p} t ; \vec{p}^{\prime} t^{\prime}\right)=<T a_{\vec{p}}(t) b_{\vec{p}^{\prime}}\left(t^{\prime}\right)>$

From equation of motion for Green functions $G_{a}\left(\vec{p} t ; \vec{p}^{\prime} t^{\prime}\right)$ and $F^{+}\left(\vec{p} t ; \vec{p}^{\prime} t^{\prime}\right)$ in $p, \omega$-representation, their poles may be obtained. Their equality to zero give spectrum of the system taking into account the effect of the electromagnetic radiation of the semiconductor, namely

$\omega_{1,2}=\frac{1}{2}\left[\xi_{c}(\vec{p})-\xi_{h}(\vec{p})\right] \pm K$,

where $K=\sqrt{\left[\frac{\xi_{c}(\vec{p})+\xi_{h}(\vec{p})}{2}\right]^{2}+|\lambda|^{2}}$.

Taking into account (1), (2), the spectrum takes the form:

$\omega_{1,2}=\left\lfloor\alpha_{0} p_{\perp}^{2}-\beta_{0}(1-\cos z)\right\rfloor \pm$

$\pm \sqrt{\left[\alpha_{1} p_{\perp}^{2}+\beta_{1}(1-\cos z)\right]^{2}+|\lambda|^{2}}$

where

$\begin{array}{ll}\alpha_{0}=\frac{1}{2}\left(\alpha_{c}-\alpha_{h}\right), & \alpha_{h}=\frac{1}{2}\left(\alpha_{c}+\alpha_{h}\right), \\ \beta_{0}=\frac{1}{2}\left(\beta_{c}-\beta_{h}\right), & \beta_{h}=\frac{1}{2}\left(\beta_{c}+\beta_{h}\right) .\end{array}$

Thus bands (13) will be separated by gap $2 \lambda$, which may be interpreted as electron-hole pair with the formation energy $2 \lambda$.

Roots (12) were analyzed in [1] for semiconductors with the parabolic dispersion law of their carriers. It was shown that at noncoincident effective masses the carrier parabolic bands (Fig. 1a) after applying electromagnetic field change their topology, namely: there is a gap $\sim \lambda$, and the band extreme are realized in another points (see Fig. 1b).

\section{Results and discussion}

Let us analyzed the spectrum reconstruction caused by the intense electromagnetic field in the layer semiconductor case. Consider an extremal problem of $\omega_{1,2}$ from (13) with regard to $p_{\perp}$ and $z$. For the upper band, $\omega_{1}$, 
B.A. Lukiyanets: Change of carrier spectrum in layer semiconductors ...
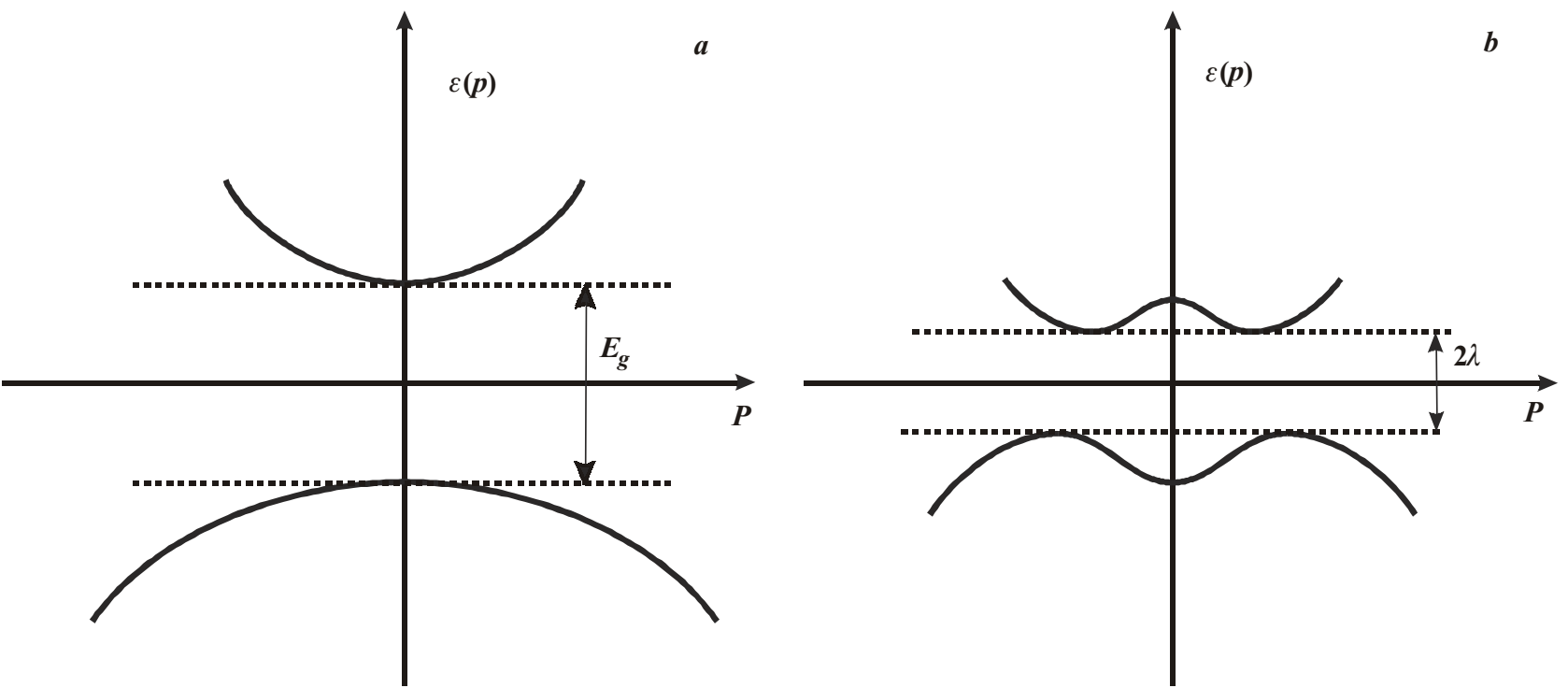

Fig. 1. Electron spectrum of an isotropic semiconductor ( $E_{g}$ is the forbidden gap) (a) and one of its possible reconstruction caused by strong electromagnetic wave $(b)$.

$$
\begin{aligned}
& \frac{\partial \omega_{1}}{\partial p_{\perp}}=2 p_{\perp}\left\{\alpha_{0}+\frac{\alpha_{1}}{K}\left[\alpha_{1} p_{\perp}^{2}+\beta_{1}(1-\cos z)-\mu_{0}\right]\right\}=0 \\
& \frac{\partial \omega_{1}}{\partial z}=\sin z\left\{\beta_{0}+\frac{\beta_{1}}{K}\left[\alpha_{1} p_{\perp}^{2}+\beta_{1}(1-\cos z)-\mu_{0}\right]\right\}=0
\end{aligned}
$$

(14), (15) are simultaneous equations, which may be divided on the subsystems:

a) $\left\{\begin{array}{c}\vec{p}_{\perp}=0 \\ \sin z=0\end{array}\right.$

From (16) it follows that critical point (in variables $\left.\left(\vec{p}_{\perp}, z\right)\right)$ is $(0,0)$.

b) $\left\{\begin{array}{c}\vec{p}_{\perp}=0 \\ \beta_{0}+\frac{\beta_{1}}{K}\left[\alpha_{1} p_{\perp}^{2}+\beta_{1}(1-\cos z)-\mu_{0}\right]=0\end{array}\right.$

and critical point is $(0, A)$, where

$$
A=\frac{\beta_{0}|\lambda|}{\sqrt{\beta_{1}^{2}-\beta_{0}^{2}}} .
$$

c) $\left\{\begin{array}{c}\alpha_{0}+\frac{\alpha_{1}}{K}\left(\alpha_{1} p_{\perp}^{2}+\beta_{1}(1-\cos z)-\mu_{0}\right)=0 \\ \sin z=0\end{array}\right.$

Here, the critical point is $\left( \pm \sqrt{\frac{\mu_{0}-B}{\alpha_{1}}}, 0\right)$, where

$B=\frac{\alpha_{0}|\lambda|}{\sqrt{\alpha_{1}^{2}-\alpha_{0}^{2}}}$.
And at last the simultaneous equations

$$
\left\{\begin{array}{l}
K+\frac{\alpha_{1}}{\alpha_{0}}\left(\alpha_{1} p_{\perp}^{2}+\beta_{1}(1-\cos z)-\mu_{0}\right)=0 \\
K+\frac{\beta_{1}}{\beta_{0}}\left(\alpha_{1} p_{\perp}^{2}+\beta_{1}(1-\cos z)-\mu_{0}\right)=0
\end{array}\right.
$$

are inconsistent ones.

In the case b) $z$ is real on condition that

$\left|\frac{A}{\beta_{1}}-\beta_{1}-\mu_{0}\right| \leq 1$ only or

$A \leq \mu_{0} \leq A+2 \beta_{1}$,

and $\vec{p}_{\perp}$ is real (see case c) at

$\mu_{0} \geq B$.

Thus, at various value intensity of the electromagnetic wave intensity, $\mu_{0}=\frac{\Omega-E_{g}}{2}$, it may be qualitatively different reconstruction of the carriers spectrum or topology of their isoenergetic surface.

Let us $A>B$ case, i.e. $\frac{\beta_{0}}{\sqrt{\beta_{1}^{2}-\beta_{0}^{2}}}>\frac{\alpha_{0}}{\sqrt{\alpha_{1}^{2}-\alpha_{0}^{2}}}$ or $\frac{\beta_{c}-\beta_{h}}{\beta_{c} \beta_{h}}>\frac{\alpha_{c}-\alpha_{h}}{\alpha_{c} \alpha_{h}}$ (It should be noted that in spite of the fact that itself $\alpha_{c}, \alpha_{h}>\beta_{c}, \beta_{h}$, the last inequality or opposite one are possible as long as there are not only values $\alpha_{c}, \alpha_{h}, \beta_{c}, \beta_{h}$, but their differences). 


\section{B.A. Lukiyanets: Change of carrier spectrum in layer semiconductors ...}

Case i). Let's $B<A$. Then the spectrum reconstruction is presented graphically as

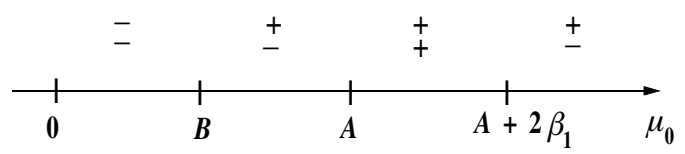

Here sign $+(-)$ means possibility (absence) of such reconstruction. The upper sign is connected with variable $\vec{p}_{\perp}$, and lower one with $z$.

Case ii). $A<B<A+2 \beta_{1}$. Then

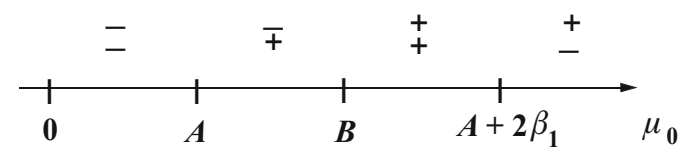

Case iii). $B>A+2 \beta_{1}$. At that

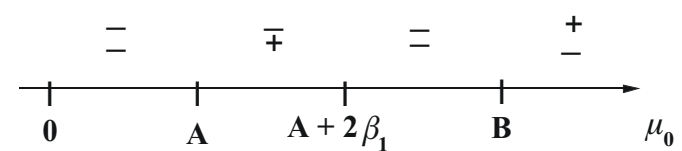

We may analyze the lower root of (13), $\omega_{2}$. In this case its qualitative change will be the same.

\section{Conclusions}

Obtained results allow to draw some conclusions. In particular:

The spectrum reconstruction of Fig. 1b type is possible starting with some intensity of the electromagnetic wave, namely $\mu_{0}>B$ (i) or $\mu_{0}>A$ (iii). Furthermore, simultaneous reconstruction both in $\left(p_{x} p_{y}\right)$ plane and along $z$ takes place at $\mu_{0}$ from $\left[A, A+2 \beta_{1}\right]$ (i) and $\left[B, A+2 \beta_{1}\right]$ (ii) only. In case (iii) such situation impossible at any $\mu_{0}$ (and consequently and $\Omega$ ).

There are known simple external factors to change electron interlayer mixing $\beta_{c}, \beta_{h}$ in the layer semiconductors (f. ex., hydrostatic or $\mathrm{C}$-axis pressure). By these factors may rule over topology of the energetic surfaces in the layer crystals in the field of the intense electromagnetic radiation.

Existence of presented limitations on the spectrum reconstruction in the layer semiconductors substantially differs from analogous reconstruction in excitonic dielectric or superconductor with the parabolic dispersion law of carriers [1].

\section{References}

1. V.M.Galitskij, V.F.Elesin. Resonanse Interaction of Elelctromagnetic Field with Semiconductors. Energoizdat. Moscow. 1986 (in Russian)

2. R.C.Fivaz, Ph.E.Schmid. Transport Properties of Layered Semiconductors. In Optical snd Electrical Properties, Ed.P.A.Lee, pp.343-384, D.Reidel Publ.Co, DordrechtHolland (1978).

3. V.M.Galitskij, S.P.Goreslavskij, V.F.Elesin. Electrical and Magnetic Properties of a Semiconductor in Field of Strong Electromagnetic Wave// Zh.Exper.and Teor.Phys, - 57(1), pp.207-217 (1969) (in Russian).

4. Yu.V.Kopayev. On Theory of Interrelation Electron and Structure Transformation in Superconductors. In Some Problems of Superconductivity (Papers of Lebedev's Physical Institute), Nauka, Moscow, pp.3-100 (1975) (in Russian). 